\title{
Tsafon
}

Revue d'études juives du Nord

$76 \mid 2018$

Expressions yiddish de la nature

\section{Un bouleau à Yad Eliyahu. La nature chez Binyomin Heller}

\section{Benny Mer}

\section{(2) OpenEdition}

1 Journals

Édition électronique

URL : https://journals.openedition.org/tsafon/1347

DOI : $10.4000 /$ tsafon. 1347

ISSN : 2609-6420

Éditeur

Association Jean-Marie Delmaire

Édition imprimée

Date de publication : 1 décembre 2018

Pagination : 113-122

ISSN : 1149-6630

\section{Référence électronique}

Benny Mer, « Un bouleau à Yad Eliyahu. La nature chez Binyomin Heller », Tsafon [En ligne], 76| 2018,

mis en ligne le 30 juin 2019, consulté le 25 juin 2021. URL : http://journals.openedition.org/tsafon/

1347 ; DOl : https://doi.org/10.4000/tsafon.1347

Tsafon. Revues d'études juives du Nord 


\title{
Un bouleau à Yad Eliyahu La nature chez Binyomin Heller
}

Benny Mer*

\begin{abstract}
À l'instar de nombreux lecteurs, j'ai fait la connaissance de Binyomin (Binem) Heller (1908-1998) à travers le poème «Mayn shvester Khaye »[Ma sœur Khaye], texte délicieux dans lequel une fillette de dix ans aux yeux verts et aux cheveux bruns élève ses petits frères et sœurs dans un immeuble de la rue Smocza à Varsovie, leur donne à manger, les coiffe et leur chante des berceuses. Mais ce poème, en apparence nostalgique, se transforme finalement en saga cruelle : en effet, Khaye a été assassinée à Treblinka «et dans l'État d'Israël, [le poète est] le tout dernier à l'avoir connue ${ }^{1}$. Ce poème, tiré du recueil Zey veln oyfshteyn [Ils se révolteront] ${ }^{2}$ publié par Heller en 1984 est précisément celui qui m'a incité à m'insurger contre l'oubli. J'ai voulu connaître Khaye, sauver ne fût-ce que son souvenir. " Celui qui meurt est condamné à être oublié des cœurs $»^{3}$ dit le Talmud. Sauf qu'il n'y avait rien de naturel dans cette mort-là, de sorte que la sentence talmudique ne peut s'appliquer ni à Khaye ni à ses petits voisins. Aussi suis-je parti

\footnotetext{
* Éditeur chez Magnes Press, enseignant de yiddish à Beyt Sholem Aleykhem, Tel Aviv, rédacteur en chef de la revue en langue Davke. Texte traduit de l'hébreu par Michèle Tauber.

${ }^{1}$ " Mayn shvester Khaye» [Ma sœur Khaye], Zey veln oyfshteyn, Tel Aviv, Farlag Y. L. Peretz, 1984, p. $26:$ «Un ikh bin in der yiddisher medine / der same letster, vos hot zi gekent $»$.

${ }^{2}$ Ibid., p. 24-25.

${ }^{3}$ Traité Pesahim, 54a.
} 
enquêter sur cette rue Smocza qui a été complètement détruite et effacée par la guerre. Certes, il existe aujourd'hui à Varsovie une rue qui porte le même nom ; pour autant, pas une seule pierre n'avait subsisté, pas un seul de ses habitants d'alors n'était demeuré pour tenter de la reconstruire, ne serait-ce que par les mots. Ainsi sont revenus à la surface des noms oubliés et des récits momifiés dans des journaux et des Yizker bikher [livres du souvenir]. C'est grâce à eux que j'ai pu dessiner la carte urbaine d'une culture disparue, une cartographie unique et particulière et en même temps si semblable à celle des rues voisines, dans le quartier presque entièrement juif de Muranów.

\section{Enfance}

Dans la poésie de Binem Heller, la rue Smocza figure en tant qu'adresse réelle en même temps que symbolique. Bien que, dans sa jeunesse, sa famille ait déménagé dans le quartier de Praga à Varsovie, tout au long de son œuvre, qui s'étale sur près de soixante ans, Heller ne cesse de revenir sur la rue de son enfance. Dès 1933, il insiste sur sa spécificité dans le poème «Smocza mayn hoyz » [Smocza mon foyer] en décrivant les cours intérieures de cette rue où ne passaient ni l'air ni la lumière : «Seulement quelques miettes d'air et de lumière / dans une cour rectangulaire ?» ${ }^{4}$. Cependant, les enfants grandissent en s'abreuvant d'histoires dans lesquelles un brigand sort de la forêt, le lieu le plus sauvage et a priori le plus éloigné de la tradition juive.

Dans un autre poème, plus tardif, Heller écrit : « Mes origines sont habituées aux pierres et aux briques / Mon arrière-grand-père vivait déjà à Varsovie ${ }^{5}$. En effet, Heller est un poète varsovien, un poète de la ville par excellence, qui s'identifie aux paysages urbains qu'il décrit. Mais sa poésie regorge néanmoins de découvertes et d'expressions ayant trait à la nature. Quelle fonction un poète de l'urbanité comme Binem Heller attribue-t-il à la nature? Quelles transformations induit le départ de Varsovie pour Tel Aviv? S'agit-il d'un usage secondaire, « instrumentalisé » par le poète, et qui pourrait nous renseigner sur la conception de la nature à l'époque où Heller composait sa poésie?

\footnotetext{
${ }^{4}$ Dos ershte lid [Le premier poème], Varshe, Farlag Yidish bukh, 1956, p. 28-29 : « Iz vozhe, az likht un az luft iz gor knap do, / in kantikgemoyertn hoyf? ».

5 «Fun vanen» [D'où ?], Klorkeyt [Clarté], Varshe, Farlag Yidish bukh, 1957, p. 141 : «Mayn shtam iz tsu shtayner un tsigl gevoynt. / Mayn urzeyde hot shoyn in Varshe gevoynt».
} 


\section{Poésie et idéologie}

Les premières poésies de Binem Heller, composées en 1931, expriment sa foi dans le communisme, décrivent le combat des ouvriers et la lutte de classes. Plus que le blé, c'est le pain qui est présent; plus que les champs figurent les usines. Dans le poème daté de 1932 intitulé "Sheyn iz der poylisher harbst» [Beau est l'automne polonais], il dépeint les meules de foin, la chute des feuilles qui l'enchantent. Mais l'automne reste avant tout synonyme de la tuberculose contractée par un camarade (du parti bien sûr) et des poings qui se serrent dans le froid : la beauté de l'automne se gausse en réalité de l'ouvrier démuni et Heller lui renvoie cette image avec ironie. Dans « Harbstlid» [Poème d'automne] ${ }^{6}$ composé en 1934, l'automne est loin d'apparaître dans toute sa splendeur dans un écrin naturel. Il s'agit en fait de l'automne urbain en gris et noir, associé au bruit de la pluie, aux rafales de vent et aux soupirs larmoyants du chômeur qui fait les cent pas. Dans « Mayn gortn » [Mon jardin] $]^{7}$, le poète décrit un rêve qui se déroule dans des vergers de pommiers et d'orangers. Mais là encore, le pain et le statut social apparaissent en définitive plus importants que les fruits de l'imagination.

Les saisons sont en fait absentes du temps urbain chez Heller : le poète ne parvient même pas à distinguer le printemps, symbolisé par la rosée sur les lilas, car il vit dans la prison et dans la réalité de l'injustice, aux antipodes d'une nature libre. Heller récuse non seulement la description idyllique de la nature mais aussi de façon implicite les symboles les plus caractéristiques de la poésie nationale sur la nature, en l'occurrence la poésie polonaise. De même qu'un Juif pratiquant est censé détourner les yeux du spectacle de la nature - «Celui qui, en voyageant, médite la Loi et interrompt sa méditation pour s'écrier : Que cet arbre est beau! Que ce champ est bien cultivé ! Celui-là, selon l'Écriture, compromet sa vie ${ }^{8}$, de la même façon, un communiste orthodoxe se doit de réfuter la beauté du lilas, cette fleur si populaire en Pologne.

Mais il arrive aussi que, succombant à la nostalgie du printemps, Heller renonce à la tonalité sarcastique de sa poésie. Dans un long poème composé en 1936, «Tsu a nayem friling » [À un printemps nouveau], il décrit le sommeil hivernal, long et rigoureux, dans lequel la ville est

\footnotetext{
${ }^{6}$ Ibid., p. 45.

${ }^{7}$ Ibid., p. 40.

${ }^{8}$ Traité Pirkei Avot [Maximes des Pères], 3-7, trad. Moïse Bruhl, Colbo, Paris, 1986.
} 
plongée: les gens rêvent de pain, mot qui rime, en yiddish, avec [le drapeau] rouge : "Le beau rêve que je fais en rêvant de pain [broyt] / aime beaucoup rimer avec le rouge $[$ royt $]$ / d'une étoile rouge et d'un drapeau rouge ${ }^{9}$. Ils rêvent aussi de lutte et de solitude, en particulier celle des vendeurs de beygelekh [petits pains], des prostituées et des ivrognes. Malgré tout, il décrit un geste religieux - panthéiste - lorsqu'il se prosterne devant le printemps : «Et lorsque la première hirondelle arrive à tire-d'aile, / d'inquiétude nous nous prosternons ${ }^{10}$. Heller utilise le printemps comme un symbole, pas uniquement comme l'annonce d'un avenir socialiste radieux mais aussi comme l'image bien connue de la jeunesse, " [sa] jeunesse, comme un tout jeune rameau de lilas $»{ }^{11}$. Il a besoin d'un long poème pour faire sentir la longueur de l'hiver et l'arrivée inattendue du printemps. Finalement, le soleil est là, mais même alors, « il sourit, issu d'une larme, / les yeux grand ouverts, il brille avec insolence $»^{12}$. Dans les derniers vers du poème, le printemps arrive de l'extérieur, de la forêt et des champs alentour, et il est le porteur d'une nouvelle, d'un autre lieu et d'un autre temps, celui de l'avenir : «Avec les arbres de la forêt et l'herbe des champs / le printemps a assiégé la ville et a tout recouvert. / Et moi qui suis aussi son soldat je chante à présent son poème! ${ }^{13}$. On remarquera que dans ce poème engagé, Heller juge bon d'écrire qu'il accueille le printemps en yiddish : « J'occuperai la terre entière avec un chant / et justement avec le yiddish de mon poème ! ${ }^{14}$. Ce vers où il choisit d'être à la fois communiste et yiddishiste $^{15}$ est particulièrement éloquent et important pour l'œuvre future du poète.

\footnotetext{
${ }^{9}$ Klorkeyt [Clarté], op. cit., p. 78 : «Der sheyner kholem mayner vegn broyt / hot zeyer lib tsu gramen zikh mit royt / fun roytn shtern un fun royter fon ».

${ }^{10}$ Ibid., p. 71 : «Un az di ershte shvalb kumt on fun fli / fun umru beygn mir zikh in di kni ».

${ }^{11}$ Ibid., p. 72 : « Mayn yugnt, vi a tsveygl frisher bez ».

${ }^{12}$ Ibid., p. 78 : «Zi shmaykhlt antkegn aroys fun a trer, / in ofene oygn mit khutspe zi glit».

${ }^{13} I d$., « Mit beymer fun vald un mit grozn fun feld / hot friling balagert di shtot un bashtelt. / Oykh ikh bin zayn zelner un zing itst zayn lid ».

${ }^{14}$ Ibid., p. 71 : «Ikh vel di erd farnemen mit gezang / un davke mit dem yiddish fun mayn lid $\gg$.

${ }^{15}$ yiddishisme : mouvement né à la fin du XIX ${ }^{\mathrm{e}}$ siècle en Russie et qui prône l'usage du yiddish comme l'expression authentique de l'esprit de la nation juive. Dans l'entre-deux guerres, le yiddishisme est associé à un nationalisme diasporique, les valeurs yiddishistes étant l'usage du yiddish comme langue vernaculaire et littéraire ainsi que l'adhésion à l'idéal socialiste.
} 
À cause de son activité communiste, Heller est contraint de quitter la Pologne pour la France et la Belgique (où vivaient alors ses deux frères). Dans le poème «A kholem » [Un rêve] composé à Paris en 1938, la nostalgie pour son pays est illustrée par l'un de ses symboles les plus éclatants: la Vistule, le fleuve qui traverse toute la Pologne, et qui, chemin faisant, " à celui qu'elle rencontre en chemin / le vent, la forêt, le ciel et la prairie / [...] demande des nouvelles d'un voisin de la cour de la rue Smocza / qui s'est fourvoyé de par le vaste monde ${ }^{16}$. Précisons que tout au long de son œuvre poétique classique, Binem Heller utilise le mètre rimé, quitte à créer parfois quelques discordances. Ainsi, il fait rimer hoyf, "la cour », statique, avec loyf, " en chemin ", pour signifier la Vistule toujours en mouvement. Mais par ailleurs, c'est bel et bien l'exil qui éveille la nostalgie du poète et l'usage qu'il fait des symboles nationaux de la nature polonaise n'est pas fortuit. Il se réfère à d'autres Polonais en exil avant lui, au premier rang desquels Juliusz Słowacki (1809-1849) et s'inscrit en cela, dans le sillon d'un autre poète yiddish, Avrom Sutzkever (1913-2010) et du long poème ${ }^{17}$ où celui-ci se sépare de son pays natal en 1946. En exil, Heller n'est pas seulement un poète révolutionnaire mais aussi un romantique polonais qui n'hésite pas à introduire des éléments nationaux fondamentaux dans la poésie de la nature. En ce sens, si Heller est incontestablement un poète communiste, il est également un poète polonais et juif.

\section{Poésie et Shoah}

Dans la poésie de Heller sur la Shoah écrite pendant la guerre alors qu'il s'était exilé en URSS, la nature est plus d'une fois symbole de trahison. C'est un motif très répandu, que l'on retrouve entre autres dans le long poème de Hayim Nahman Bialik, «Be-'ir ha-harega » [Dans la ville du massacre] : "Le soleil dardait, l'acacia fleurissait et l'égorgeur égorgeait ${ }^{18}$. De même, le long poème de Heller intitulé "Varshe $1939 »$ [Varsovie 1939] s'ouvre sur une description idyllique des saisons

\footnotetext{
${ }^{16}$ Klorkeyt [Clarté], op. cit., p. 90 : « Nor vemen zi bagegnt in ir loyf, / dem vint, dem vald, dem himl un di lonke / zi fregt zikh oyf a mentsh fun smotsher hoyf / vos hot zikh oyf der groyser erd farblonket $»$.

${ }^{17}$ Tsu Poyln, [À la Pologne], New York, Matones, 1948, p. 155.

${ }^{18}$ «Be-'ir ha-harega » [Dans la ville du massacre], Shirim [Poèmes] Or Yehuda, Devir, 2004, p. 254 : «Ha-shemesh zarha, ha-shita parha, ve-ha-shohet shahat». Suite au pogrome de Kishinev en 1903, Bialik compose ce poème en hébreu avant de le traduire en yiddish.
} 
polonaises où alternent l'été porteur de pain et de moisson, le printemps qui s'épanouit (pour le poète !) et ainsi de suite : «l'hiver polonais est beau / l'automne répand et disperse ses ors $»{ }^{19}$. Heller multiplie les détails dans la description de la nature pour insister sur l'invasion contrenature de la Pologne par les nazis. Les armes et les avions de combat s'opposent à la nature et le feu qui embrase les champs mûrs de la Pologne souligne l'infériorité et la défaite de la nature.

En 1939 à Białystok, dans le poème «Ikh bin do » [Je suis là], Heller réutilise la nature de façon symbolique par cette métaphore à la fois triste et belle : «Il tombe des feuilles-morceaux-d'étoffe jaunies » ${ }^{20}$. Les feuilles d'automne symbolisent la fin toute proche, c'est-à-dire la nature qui collabore avec l'ennemi. L'automne polonais gorgé d'or se transforme en marque de l'ignominie juive. Il n'est donc pas étonnant que Heller tourne le dos à la nature polonaise en particulier. Dans le poème « Tsum flier » [À l'aviateur] écrit également à Białystok en 1940, il se refuse à reconnaître la splendeur de l'aigle et lui préfère les ailes d'acier d'un avion de combat.

En 1948, Heller décide de quitter l'Union Soviétique (et Moscou où il a vécu quelques années) et retourne en Pologne dans l'intention de prendre à rebours la mesure du temps juif. La plupart des Juifs revenus en Pologne après la guerre en sont repartis et le poème de Sutzkever « Tsu Poyln » [À la Pologne $]^{21}$ rythme la marche de ce mouvement. Ce poème est en effet une sorte de règlement de comptes avec la Pologne, dont les principaux représentants sont les poètes et la nature merveilleuse peuplée de forêts, prairies, cigognes, etc. Mais Heller est l'un des seuls à avoir choisi à la fois de demeurer en Pologne communiste et de rester attaché à la création littéraire en yiddish. Son recueil de poèmes publié en 1950 porte le titre audacieux de Friling in Poyln [Printemps en Pologne] et, de fait, il est tout entier consacré à la nature polonaise qui s'épanouit de nouveau après la Shoah. Le recueil s'ouvre sur la description d'une fuite polysémique vers la forêt : la fuite de 1939 lors de l'invasion de la Pologne, et la fuite des romantiques hors de la ville vers la nature. Là, le poète voit le sapin, à la fois réel et symbolique, demeuré verdoyant durant tout l'hiver. Le recueil suivant publié en 1951 porte le titre tout aussi engagé de Heymerd [Ma terrefoyer]. Dans l'un des premiers

\footnotetext{
${ }^{19}$ Tiré du recueil Durkhn shotn [À travers l'ombre], Lodsh, Farlag Yidish bukh, 1948, p. 5 : « Der vinter in Poyln iz sheyn, / der harbst varft mit gold zikh un shit».

${ }^{20}$ Ibid., p. 42 : «S'faln gele late bleter».

${ }^{21} \mathrm{Cf}$. note 17 .
} 
poèmes, «Umker » [Retour], Heller dépeint la solitude du poète yiddish dans la nouvelle Varsovie, celle d'après-guerre : «Non, cela ne me fait pas souffrir de voir ces pierres tordues / qu'on a brisées après les avoir prises au Vieux Pont. / Cela me fait seulement mal de ne rencontrer ici personne / de mes vieux amis avec qui je puisse échanger un salut ${ }^{22}$. Dans un autre poème ${ }^{23}$, il décrit la sensation d'étrangeté ressentie vis-àvis de ses nouveaux voisins qui prennent de plus en plus de distance visà-vis de la Varsovie juive d'antan.

Il apparaît que seule la nature polonaise, demeurée semblable à ce qu'elle était avant la guerre, crée la sensation du heymish, la sensation d'être à la maison, dans un pays qui a changé de visage. Heller est toujours en quête de symboles pour incarner la réalité : le printemps commence à s'épanouir en avril, le mois des héros de la révolte du ghetto de Varsovie, et le «merveilleux mois de mai » est consacré à la victoire sur les nazis : « Le printemps célèbre le mois d'avril comme sa victoire et accroche un drapeau rouge aux fenêtres $»^{24}$. Heller admire la nature avec une ferveur quasi fanatique : "Je ne suis pas religieux - mais je pourrais me prosterner devant un printemps en fleurs $\gg{ }^{25}$.

Dans le recueil qui suit, intitulé In undzer tsayt [De notre temps] et publié en 1954, Heller continue à s'identifier avec la nature polonaise : "Si je devais me réincarner en arbre / je voudrais être un bouleau ${ }^{26}$. Heller choisit l'arbre le plus typiquement polonais, berioze, le bouleau, et dans le même temps, il situe son poème à l'automne, lors de la chute des feuilles, en pleine tempête : "Alors dans une explosion de force et de nostalgie / de ce qui fut je me souviendrai ${ }^{27}$. Également dans le long poème "Yidn in Poyln» [Juifs en Pologne], qui est peut-être une réponse au poème «Tsu Poyln» de Sutzkever, Heller associe le bruissement le plus typiquement juif qui soit, l'étude d'une page du Talmud, au brouhaha de la forêt polonaise : « La forêt qui bruisse sur des

\footnotetext{
${ }^{22}$ Heymerd, Varshe, Farlag Yidish bukh, 1951, p. 6: « Nayn, s'tut nisht vey far yene krume shteyner, / vos m'hot fun altn bruk aroysgerisn. / mir tut bloyz vey, vos s'treft mir do nisht keyner / fun alte fraynt / vos zol zikh mit mir grisn ».

${ }^{23}$ «In der nayer shtot [Dans la ville nouvelle], ibid., p. 21.

24 «Nitsokhn» [Victoire], ibid., p. 98 : «Dem friling fayert er vi zayn nitsokhn / un hengt aroys di royte fon $»$.

${ }^{25}$ Ibid., p. 107 : « Ikh bin nisht frum nor in dem friling bli / volt ikh avkgeshtelt zikh oyf der kni ».

26 «Oyb farvandlt tsu vern» [Si je devais me réincarner], In undzer tsayt, Varshe, Farlag Yidish bukh, 1954, p. 14 : «Oyb farvandlt tsu vern darf ikh in a boym / vil ikh zayn a berioze ».

${ }^{27} I d$. : « Kh vel demolt in oysbrukh fun benkshaft un kraft / dem amol zikh dermonen ».
} 
centaines de lieues / a aussi l'habitude de s'abandonner au balancement de la prière avec délectation / à la question psalmodiée d'un Juif / et de lui répondre avec le nign, la mélodie talmudique ${ }^{28}$. Ce poème illustre bien la démarche de Heller à la recherche des fondements du judaïsme dans cette Pologne nouvelle. Le quotidien communiste qui l'entoure rend son «nationalisme yiddish» plus manifeste encore. Ce qui justifie sans doute la décision drastique de quitter la Pologne et de s'installer en Israël en 1956. Dans le poème «Mayn art» [À ma manière], publié dans le recueil Klorkeyt [Clarté] en 1957 après son départ de Pologne, Heller pose la question : "Suis-je déjà le dernier des troubadours / [...] la moindre trace de moi disparaîtrait-elle / justement ici, où chaque arbre m'a reconnu? ${ }^{29}$. Il tente $d$ 'y répondre dans le même poème en affirmant qu'il ne quittera pas la Pologne, cette terre où les Juifs ont vécu pendant mille ans, mais sa première rencontre avec Israël le conduit à changer immédiatement d'avis. La nature est la seule chose qui soit restée en Pologne au poète yiddish et lorsqu'il découvre que la nature de la terre d'Israël lui est familière et amicale, il consent à quitter son pays natal.

\section{Poésie yiddish et terre d'Israël}

Le premier recueil de poèmes composé par Heller en Israël, et publié à Jérusalem en 1957, s'intitule Baym rand [À la lisière]. Dans un des poèmes qui le compose, il décrit la première nuit passée dans son nouveau pays. Contrairement au célèbre poème de Sutzkever sur sa première nuit à Tel Aviv, où celui-ci fait la rencontre de Natan Alterman et d'autres poètes hébreux dans un café de la ville, Heller, lui, fait connaissance avec la nature locale. C'est une nuit chaude et humide et l'obscurité ne laisse rien deviner. Malgré tout, la sensation éprouvée est univoque : « Je fais partie de la famille, la terre / m'a aussi reconnu dans la nuit ${ }^{30}$. Ici, nul besoin des mille ans d'histoire : il se sent « de la famille » dès la première nuit. Les jours suivants le mettent en contact avec la nature israélienne, évoquée à travers la rudesse des collines

\footnotetext{
${ }^{28}$ Ibid., p. 56 : « Der vald vos roysht avek oyf hundert meyln, / flegt oykhet in metikes zikh tsevign, / un oyf a yidns zigendiker shayle / geentfert im mit a gemore nign ».

${ }^{29}$ Klorkeyt, Varshe, Farlag Yidish bukh, 1957, p. 97 : « Tsi bin ikh shoyn der letster fun di zinger / ...tsi vet fun mir der mindster shpur farshvindn / ot do, vu yeder boym hot mir derkent?».

30 «Nakht» [Nuit], Baym rand [À la lisière], Jérusalem, Kiryat Sefer, 1957, p. 158 : « An eygener bin ikh, di erd / hot oykh in der nakht mikh derkent ».
} 
caillouteuses mais aussi la sérénité de la vallée, et il se rend compte de l'occasion unique qui s'offre à lui de renouveler son existence et sa poésie. Dans ce premier cycle, il écrit qu'il est peut-être destiné à ce qu'Israël soit le seul endroit « où [il] trouvera la valeur / de l'action et de la force dans [ses] mots $\gg{ }^{31}$. La vue de la terre limoneuse et rouge de Hertzliya l'enthousiasme au point d'éveiller en lui la tentation de renoncer au drapeau rouge. De fait, en Israël, de communiste qu'il était, Heller devient sioniste, et la nature se transforme dans ce sens.

Le poète est conscient de cette nature nouvelle qui l'entoure mais il semble toujours en quête de quelque chose de familier: un arbre à la place d'un arbre, le Yarkon à la place de la Vistule. Heller ne procède pas comme Sutzkever qui a transformé du tout au tout sa poésie sibérienne en paysages désertiques du Neguev. Chez Heller, par exemple, le mois du printemps n'est plus le mois de mai mais le mois d'Adar ${ }^{32}$, et il est lié à la tradition populaire selon laquelle les oiseaux chantent des poèmes à ce moment-là. Un sentiment d'intense émotion religieuse s'empare à nouveau de lui devant le printemps, mais cette fois il évoque un symbole exotique, de ceux que l'on rencontre aussi dans la poésie yiddish (par exemple les raisins secs et les amandes), le symbole méditerranéen de la terre d'Israël : «Der mandelboym blit» [L'amandier est en fleurs] : «Combien il est difficile aujourd'hui de n'être pas religieux / par un tel matin de mars : / mais avant de regarder autour de moi / l'amandier est déjà en fleurs $»{ }^{33}$. Le poète ignore le nom des arbres aux efflorescences bleues dans son jardin, mais ils lui rappellent le lilas. Dans le recueil Bikhides [Seul avec soi-même] paru en 1975, il trouve même un substitut au parfum enivrant du lilas : la floraison des orangers en tout lieu lui procure un fort sentiment d'exaltation ${ }^{34}$.

Or, dans le recueil Zey veln oyfshteyn évoqué au début de cette étude, un tournant s'opère dans la poésie de la nature chez Binem Heller. Après s'être identifié avec la nature telle qu'elle s'offrait à lui sur place tant en Pologne qu'en Israël, le poète recherche la nature dans le temps, autrement dit les souvenirs, et parvient ainsi à établir une sorte de synthèse d'une nature israélo-polonaise. Ce tournant, qui s'apparente à

\footnotetext{
${ }^{31}$ Ibid., p. 160 : «Vu ikh vel gefinen dem vert / fun tat un fun kraft in mayn vort».

${ }^{32}$ Le Yarkon est le fleuve qui coule à Tel Aviv. Le mois d'adar correspond à la période février-mars du calendrier grégorien. Notes de la traductrice.

${ }^{33}$ Voir le recueil Dor un doyer [Filiation et continuité], Tel Aviv, Menora, 1967, p. 86 : «Vi shver iz tsu zayn haynt nisht frum / in ot aza marts inderfri : / nokh eyder ikh kuk zikh arum / der mandelboym shteyt shoyn in bli ».

${ }^{34}$ Bikhides, Tel Aviv, Farlag Y. L. Perets, 1975, p. 40.
} 
une tentative de retour dans le passé, est sans doute la part de la poésie la plus connue et la plus significative de Heller. « De quoi ai-je besoin ? Seulement de l'odeur des bois ${ }^{35}$, écrit-il dans un poème où il tente dans un geste proustien de redonner vie à ses morts par le truchement des odeurs de la forêt. Dans un autre poème, il revoit l'arbre polonais, conscient qu'il ne pourra en utiliser le bois pour son cercueil, et malgré tout « l'arbre se tient toujours dans la même position / entièrement revêtu de vert. / Son nom demeure toujours : bouleau, / ma mère m'appelle toujours : Binem $\gg{ }^{36}$. Dans un troisième poème, il demande doublement pardon au bouleau de l'avoir abandonné, et à sa nouvelle patrie de ne pouvoir oublier l'arbre polonais d'autrefois : «La haine m'a chassé de cette vallée au bord de la Vistule. / À d'autres arbres j'ai écrit des poèmes $\gg{ }^{37}$.

Dans son dernier recueil, Der gayst fun shturm [L'esprit de la tempête], composé en 1992, Heller rompt avec les espaces temporels et se retire entre ses quatre murs, dans une vision condensée de la nature urbaine, en particulier celle des couchers de soleil. Il passe ses dernières années dans une maison du quartier de Yad Eliyahu, non loin de Tel Aviv, cloîtré dans une sorte de cour fermée, semblable à celle de la rue Smocza. Aux yeux du poète, la nature, qui tout au long de son œuvre incarne une composante dynamique aux variantes infinies tout en se faisant le symbole d'idéologies diverses et de lieux nouveaux, a cessé de se renouveler. L'un de ses tout derniers poèmes composés en 1990 s'achève sur une question dont le poète ne livre pas la réponse: «Combien de pas m'est-il encore donné de faire ? / Et pour aller où ? / Combien de fois verrai-je encore / la terre s'habiller de jaune et de vert $? »^{38}$.

\footnotetext{
${ }^{35}$ « Di mame mitn kind » [La mère et l'enfant], ibid., p. $13:$ « Vos darf ikh ? Bloyz dem reyakh funem vald $»$.

${ }^{36}$ Dedikatsye [Dédicace], ibid., p. 14: « $\mathrm{Zi}$ shteyt nokh in der zelber poze, / an ongetuene in grinem. / Ir nomen iz nokh alts : berioze, / mayn mame ruft mikh alts nokh : Binem ».

${ }^{37}$ Mayn muze [Ma muse], ibid., p. 122 : « A sine hot mikh fun dem tol bay der Vaysl fartribn. / Tsu andere beymer hob ikh shoyn di lider geshribn ».

${ }^{38}$ Der gayst funem shturm, Tel Aviv, Farlag Y. L. Perets, 1992, p. 45 : «Vifl iz mir geblibn tsu geyn / un vuhin ? / Vifl mol vel ikh nokh zen / vi di erd vert gel un grin?».
} 